Errata to article on Heredity in Adalia by Miriam A. Palmer in September number.

Page 289,8 th line from the bottom should read " 8 humeralis" instead of "8 annectans."

Page 295, 12th line from the top should read "Plate XXI" instead of "Plate III."

Page 297, 6th line from the bottom should read "Fig. 3, Plate XX" instead of "Fig. 2, Plate XX."

Page 301 and 302 in Explanation of Plates, read "Plate XX" instead of "Plate II"; and read "Plate XXI" instead of "Plate III."

\title{
INDEX TO VOLUME IV.
}

abbotii, Sphecodina, 277 .

Abdominal Ganglia, 220, 227.

Nerve Tracts in, 223.

Studied in Section, 227.

Acanthaclisis, 5.

baetica, 6 .

acarivora, Mycodiplosis, 59 .

achemon, Pholus, 272, 277

Acherontia, 264, 267.

atropos, 267.

Adalia, 283, 292

annectans, 283.

humeralis, 283.

melanopleura, 283.

Adalia, Some Notes on Heredity in the Coccinellid genus, 283.

adhesa, Camptoneuromyia, 58.

aegyptiacus, Creagris, 16 .

African myrmeleonidae, Notes on, 1.

africanus, Creagris, 15 .

agilis, Eulachnus, 54.

agrifolia, Quercus, 339.

agrifoliae, Pseudococcus, 309, 310, 316, 325,327 .

agrifoliae, Synergus, 364 .

albifacies, Promachus, 155, 165, 168.

albomanicatus, Chirotonetes, 116 .

albus, Polymitarcys, 98.

alcetris, Myrmeleon, 10.

alcidice, Nemoleon, 20.

alcione, Formicaleon, 17.

aldrichii, Promachus, 165, 171.

alecto, Theretra, 272 .

allectus, Tricorythus, 115 .

alope, Erynnyis, 278.

Alphora, 128, 328.

alternatus, Siphlurus, 116.

Amelanchier, 59.

Ameletus ludens, 117.

americana, Miastor, 56.
Amorpha, 264, 270.

populi, 270 ,

Ampeloecan, 265, 272, 273.

Ampeloeca versicolor, 273.

Ampelophaga, 272.

choerilis, 278.

myron, 277.

versicolor, 278 .

Amphibolia, 128.

Amphibolips, 359.

Amphion, 265, 273.

nessus, 277.

amyntor, Ceratomia, 262.

Anabrus, 64 .

Anal Lobes, 326.

Anal Ring, Setae of the, 326

ananassi, Thecodiplosis, 58.

anderisus, Watobius, 35 .

Andricus, 346.

brunneus, 353 .

chrysolepidis, 346 .

congregatus, 347 .

crystallinus, 348 .

dasydactyli, 349 .

kingi, 350.

pacificus, 348 .

parmula, 350 .

pattersonae, 352 .

quercus-californicus, 346 .

quercus-flocci, 352 .

wiltzae, 353 .

wisliceni, 351 .

Anisia, 135.

annectans, Adalia, 283, 284, 285, 286, $287,288,289,290,291,292,293$. $294,295,296,297,298,299,300$.

annectans-humeralis, Adalia, 293.

annectans-melanopleura, Adalia, 285, 289, 292, 293, 294, 295.

antaeus, Cocytius, 277. 


\section{$2 \mathrm{BHL}$ Biodiversity Heritage Library}

1911. "Errata, Vol. IV." Annals of the Entomological Society of America 4, 495-495. https://doi.org/10.1093/aesa/4.4.495a.

View This Item Online: https://www.biodiversitylibrary.org/item/54996

DOI: https://doi.org/10.1093/aesa/4.4.495a

Permalink: https://www.biodiversitylibrary.org/partpdf/193812

\section{Holding Institution}

Smithsonian Libraries

\section{Sponsored by}

Smithsonian

\section{Copyright \& Reuse}

Copyright Status: Public domain. The BHL considers that this work is no longer under copyright protection.

This document was created from content at the Biodiversity Heritage Library, the world's largest open access digital library for biodiversity literature and archives. Visit BHL at https://www.biodiversitylibrary.org. 